\title{
Correction to: A phase III randomized trial of gantenerumab in prodromal Alzheimer's disease
}

\author{
Susanne Ostrowitzki ${ }^{1}$, Robert A. Lasser ${ }^{2}$, Ernest Dorflinger ${ }^{3}$, Philip Scheltens ${ }^{4}$, Frederik Barkhof ${ }^{4,5,6}$, \\ Tania Nikolcheva ${ }^{6}$, Elizabeth Ashford ${ }^{7}$, Sylvie Retout ${ }^{8}$, Carsten Hofmann ${ }^{8}$, Paul Delmar ${ }^{9}$, Gregory Klein ${ }^{6}$, \\ Mirjana Andjelkovic ${ }^{8}$, Bruno Dubois ${ }^{10}$, Mercè Boada ${ }^{11}$, Kaj Blennow ${ }^{11}$, Luca Santarelli ${ }^{12}$, Paulo Fontoura ${ }^{9^{*}}$ \\ and for the SCarlet RoAD Investigators
}

\section{Correction}

Following publication of the original article [1], the authors reported errors in the formatting of the table. The details of the errors are as follows:

In Table 1, the data presented do not correspond to the row title:

- The education row currently contains incorrect data.

- The weight row currently contains the years of education.

- The APOE\&4 genotype row currently contains the weight data.

In Table 1, the data should be displayed as follows:

Variable

Intention-to-treat population $(n=797)$

Placebo

$(n=266)$

Gantenerumab

$105 \mathrm{mg}$

$(n=271)$

Gantenerumab

$225 \mathrm{mg}$

$(n=260)$

Age, years, mean (SD)

$69.5(7.5)$

$70.3(7.0)$

$71.3(7.1)$

Education, years, mean (SD)

$12.6(4.3)$
$12.9(4.8)$

$12.1(4.5)$

Weight, kg, mean (SD)

$69.8(12.9)$

$70.5(13.6)$

$70.1(12.5)$

APOE 4 genotype, ${ }^{\mathrm{a}}$

\section{Author details}

${ }^{1}$ Product Development, Neuroscience, Genentech Inc., South San Francisco, CA, USA. ${ }^{2}$ MedDay Pharmaceuticals, Boston, MA, USA. ${ }^{3}$ Formerly Roche Translational \& Clinical Research Center, New York, NY, USA. ${ }^{4}$ VU University Medical Center, Amsterdam, The Netherlands. Institute of Neurology, UCL, London, UK. ${ }^{6}$ Roche Pharma Research and Early Development, NORD, Basel, Switzerland. ${ }^{7}$ Roche Products Limited, Welwyn Garden City, UK. ${ }^{8}$ Roche Pharma Research and Early Development, Clinical Pharmacology, Roche Innovation Center, Basel, Switzerland. ${ }^{9}$ Clinical Pharmacology and Bioanalytical R\&D, Pharmaceutical Sciences, Roche Pharma Research and Early Development, Roche Innovation Center Basel, Basel, Switzerland.

${ }^{10}$ Alzheimer Institute and ICM, UMR-S975, Salpêtrière University Hospital, AP-HP, Pierre and Marie Curie University, Paris, France. ${ }^{11}$ Research Center and Memory Clinic of Fundació ACE, Institut Català de Neurociències Aplicades, Barcelona, Spain. ${ }^{12}$ Formerly Roche Pharma Research and Early Development, NORD, Basel, Switzerland.

Published online: 27 September 2018

\section{Reference}

1. Ostrowitzki $\mathrm{S}$, et al. A phase III randomized trial of gantenerumab in prodromal Alzheimer's disease. Alzheimer's Research \& Therapy. 2017;9:95. https://doi.org/10.1186/s13195-017-0318-y.

* Correspondence: paulo.fontoura@roche.com

${ }^{9}$ Clinical Pharmacology and Bioanalytical R\&D, Pharmaceutical Sciences,

Roche Pharma Research and Early Development, Roche Innovation Center

Basel, Basel, Switzerland 